\title{
A novel approach to target hypoxic cancer cells via combining $\beta$-oxidation inhibitor etomoxir with radiation
}

This article was published in the following Dove Press journal: Hypoxia

\author{
Arpit Dheeraj ${ }^{1,2}$ \\ Chapla Agarwal \\ Isabel R Schlaepfer ${ }^{3}$ \\ David Raben ${ }^{4}$ \\ Rana Singh ${ }^{2}$ \\ Rajesh Agarwal' \\ Gagan Deep ${ }^{5-7}$
}

'Department of Pharmaceutical Sciences, Skaggs School of Pharmacy and Pharmaceutical Sciences, University of Colorado Anschutz Medical Campus, Aurora, CO, USA; ${ }^{2}$ School of Life Sciences, Jawaharlal Nehru University, New Delhi, India; ${ }^{3}$ Division of Medical Oncology, University of Colorado Anschutz Medical Campus, Aurora, CO, USA; ${ }^{4}$ Department of Radiation Oncology, University of Colorado Anschutz Medical Campus, Aurora, CO, USA; ${ }^{5}$ Department of Cancer Biology, Wake Forest School of Medicine, Winston-Salem, NC, USA; ${ }^{6}$ Department of Urology, Wake Forest School of Medicine, Winston-Salem, NC, USA; ${ }^{7}$ Wake Forest Baptist Comprehensive Cancer Center, Winston-Salem, NC, USA

Correspondence: Gagan Deep Wake Forest Baptist Comprehensive Medical Center, Hanes 5048, Medical Center Boulevard, Winston-Salem, North Carolina 27I57, NC, USA

$\mathrm{Tel}+\mathrm{I} 3367169363$

Fax +I 3367164480

Email gdeep@wakehealth.edu
Background: Hypoxia in tumors is associated with resistance towards various therapies including radiotherapy. In this study, we assessed if hypoxia in cancer spheres could be effectively reduced by adding etomoxir (a $\beta$-oxidation inhibitor) immediately after cell irradiation.

Methods: We employed cancer cells' sphere model to target hypoxia. Confocal imaging was used to analyze hypoxia and expression of specific biomarkers in spheres following various treatments (radiation and/or etomoxir).

Results: Etomoxir (32.5 $\mu \mathrm{M})$ treatment improved the radiation (2.5 Gy) efficacy against growth of lung adenocarcinoma H460 spheres. More importantly, radiation and etomoxir combination significantly reduced the hypoxic regions (pimonidazole + areas) in $\mathrm{H} 460$ spheres compared to either treatment alone. Also, etomoxir and radiation combination treatment reduced the protein level of biomarkers for proliferation (Ki-67 and cyclin D1), stemness (CD44) and $\beta$-oxidation (CPT1A) in H460 spheres. We observed similar efficacy of etomoxir against growth of prostate cancer LNCaP cells' spheres when combined with radiation. Further, radiation treatment strongly reduced the hypoxic regions (pimonidazole+ areas) in CPT1 knockdown LNCaP cells' spheres. Conclusions: Together, these results offer a unique approach to target hypoxia in solid tumors via combining etomoxir with radiation, thereby improving therapeutic efficacy.

Keywords: Hypoxia, radiation, $\beta$-oxidation, Etomoxir, CPT1A

\section{Introduction}

Hypoxia (low oxygen condition) in solid neoplasms is an early phenomenon, which induces genetic and epigenetic changes in cancer cells and various tumor microenvironment components leading to increased angiogenesis, stemness, metabolic alterations, and selection of resistant clones. ${ }^{1}$ Tumor hypoxia status and hypoxia-related biomarkers are associated with poor prognosis, treatment failure, and disease relapse. ${ }^{1}$ For example, Hung et al reported that higher hypoxia-inducible factor-1 alpha (HIF-1 $\alpha$ ) expression in lung cancer patients was associated with a shorter recurrence-free survival. ${ }^{2}$ Turaka et al also reported that hypoxia in prostate cancer $(\mathrm{PCa})$, that is, low mean hypoxic prostate/muscle $\mathrm{pO}_{2}$ ratio, significantly predicts poor long-term biochemical outcome. ${ }^{3}$ Milosevic et al reported that tumor hypoxia is associated with early biochemical relapse after radiotherapy and predicts local recurrence. ${ }^{4}$ Similarly, several other studies have shown that hypoxia is involved in radioresistance in various cancers. ${ }^{5-9}$ Therefore, it is important to simultaneously target hypoxia in tumors along with various therapies for effective treatment and better outcomes.

Several approaches have been tried to overcome or target hypoxia or hypoxia-induced signaling in tumors. Numerous specific or nonspecific inhibitors of HIF- $1 \alpha$ and HIF-2 $\alpha$ 
have been tested to improve the efficacy of various therapies against cancer. ${ }^{10}$ For example, YC-1 treatment reduced radiation-induced HIF-1 $\alpha$ activation and delayed tumor growth. ${ }^{11}$ Similarly, the selective inhibitor of Ataxia telangiectasia and Radd3-related protein (ATR), VE-821, increased the radiationinduced loss of cell viability under hypoxic conditions in different cancer cells. ${ }^{12}$ These studies confirm that tumor hypoxia is an important target to overcome radioresistance and to improve the therapeutic efficacy of fractionated radiation. However, one unique challenge is the delivery of these pharmacological agents (HIF-1 $\alpha$ or ATR inhibitors) to hypoxic cells as hypoxic areas have reduced blood supply and are mostly beyond the diffusion limit for drug penetrance to hypoxic core. Therefore, additional and novel measures are warranted to better target hypoxic cancer cells.

A complex metabolic reprogramming is an essential feature of cancer cells to satisfy the demand of energy and macromolecules for sustained proliferation under extreme tumor microenvironment. ${ }^{13,14}$ Especially, many facets of lipid metabolism including accumulation of lipid droplets, lipogenesis, and $\beta$-oxidation, are important in the survival and adaptation of cancer cells to low oxygen conditions. ${ }^{15-18} \mathrm{We}$ have recently reported that $\mathrm{PCa}$ cells accumulate lipids under hypoxia in association with increased HIF-1 $\alpha$, ATP-citrate lyase, and fatty acid synthase expression. ${ }^{19}$ We also reported that PCa cells rapidly used their stored lipids for proliferation following reoxygenation of hypoxic cells. Importantly, inhibition of carnitine palmitoyltransferase 1 (CPT1) by etomoxir and stable CPT1 knockdown resulted in compromised growth of hypoxic PCa cells following reoxygenation. ${ }^{19}$ These studies suggested that hypoxic cancer cells could be effectively targeted by etomoxir following reoxygenation. To evaluate this hypothesis, in the present study, we combined etomoxir with radiation treatment in a spheroid model, where radiation would reduce the sphere size and reoxygenate the hypoxic cells resulting in elimination by etomoxir treatment due to their dependence on $\beta$-oxidation for their survival. Results showed that etomoxir added to radiation could effectively reduce hypoxia and inhibit cancer cell growth in a sphere model.

\section{Methods and materials Reagents and cell culture}

Human lung epithelial carcinoma H460 and prostate carcinoma LNCaP cells were from ATCC (American Type Culture Collection, Manassas, VA, USA) and cultured in Roswell Park Memorial Institute (RPMI) 1640 medium with 10\% fetal bovine serum (FBS) and 1\% penicillin-streptomycin antibiotics. RPMI1640, FBS, DMEM/F12, penicillin-streptomycin antibiotics, trypsin $(0.25 \%$ and $0.05 \%), \mathrm{B} 27, \mathrm{~N} 2$, recombinant epidermal growth factor, fibroblast growth factor, Alexa Fluor 488/594 secondary antibodies, and gold antifade reagent with 4',6-diamidino-2-phenylindole (DAPI) were from Life technologies (Grand Island, NY, USA). Etomoxir was from Sigma Aldrich Co. (St. Louis, MO, USA). Pimonidazole was from Hypoxyprobe Inc. (Burlington, MA, USA). Primary antibody for cyclin D1 was from Cell Signaling Technologies (Beverly, MA, USA), CD44 from Santa Cruz Biotechnology (Dallas, TX, USA), CPT1A from Proteintech (Rosemont, IL, USA), and Ki-67 from Abcam (Cambridge, MA, USA).

\section{Spheroid culture assay}

H460 cells (2,500-5,000 cells/well) or LNCaP cells $(10,000$ cells per well) were seeded in ultralow attachment six-well culture plates (Corning, St. Louis, MO, USA) in stem cell media (DMEM/F12 media supplemented with B27 and N2). Additionally, for LNCaP spheroid culture, $0.5 \mathrm{~mL}$ media with recombinant EGF (20 ng/mL) and FGF $(10 \mathrm{ng} / \mathrm{mL})$ were added every $72 \mathrm{~h}$. At day 7 (or as indicated), spheres were irradiated (2.5 Gy as a single fraction dose) using an RS 2000 Biological Irradiator (Rad Source Technologies, Buford, Georgia 30518, USA). Spheres were immediately treated with etomoxir (experiment day 0 ) and every $48 \mathrm{~h}$ thereafter. At the end, sphere numbers was counted and spheres' area was measured using AxioVision Release 4.7 software. A brief summary of the experimental scheme is shown in Figure 1A.

For second-generation spheres, primary spheres were collected by brief centrifugation and incubated with trypsin $(0.05 \%)$ for $5 \mathrm{~min}$ with frequent gentle pipetting to dissociate the spheres. Once the single cells were formed, cells were counted and replated in ultralow attachment plates as described above. Cells were treated with etomoxir $32.5 \mu \mathrm{M}$ after $24 \mathrm{~h}$ of seeding and every $48 \mathrm{~h}$ thereafter. The number of spheres was counted after day 6 of treatment.

\section{Clonogenic assay}

Spheres were dissociated, counted, and seeded in 6-well plates $\left(1 \times 10^{3}\right.$ cells/well $)$ in regular culture media. Cells were treated as indicated, and at the end of day 7 , cells were fixed with $4 \%$ formalin, stained with $1 \%$ crystal violet, and colonies with $\geq 50$ cells were counted under a microscope.

\section{Hypoxia staining with pimonidazole}

Spheres were treated with pimonidazole $(200 \mu \mathrm{M})$ for $2 \mathrm{~h}$. Thereafter, spheres were centrifuged at 1,000 rpm for $5 \mathrm{~min}$ and fixed in $4 \%$ buffered formalin. Next, spheres were transferred in eight-well chamber slides coated with a thin layer of matrigel, permeabilized with PBS with Tween 20 (PBST; 
A

Experiment: day 0

H460 cells $\left(2.5 \times 10^{3}\right)$ cultured in stem-cell specific media on low attachment plates

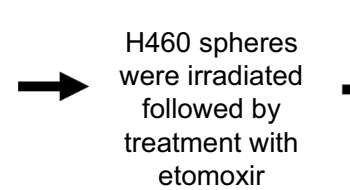

H460 spheres ere irradiated treatment with etomoxir day 2

day 4

day 6

B

H460 spheres, day 6

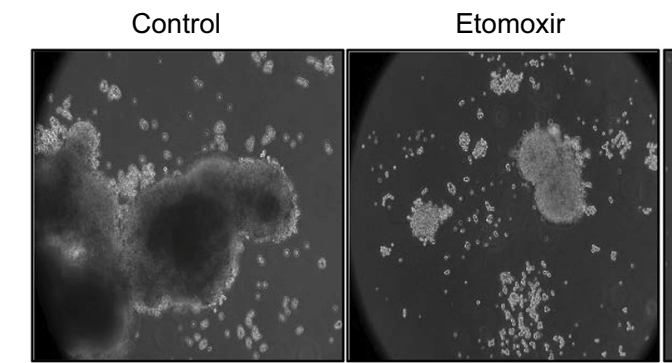

Radiation

Radiation + Etomoxir

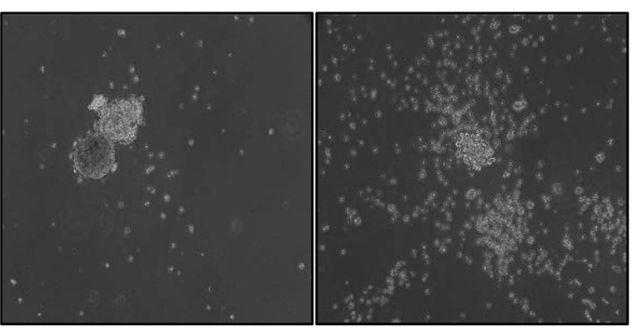

C

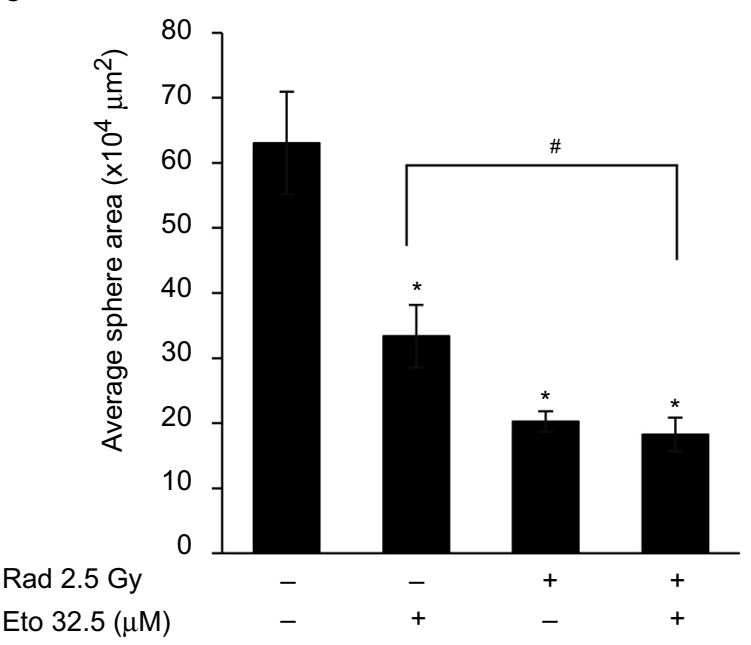

D

E
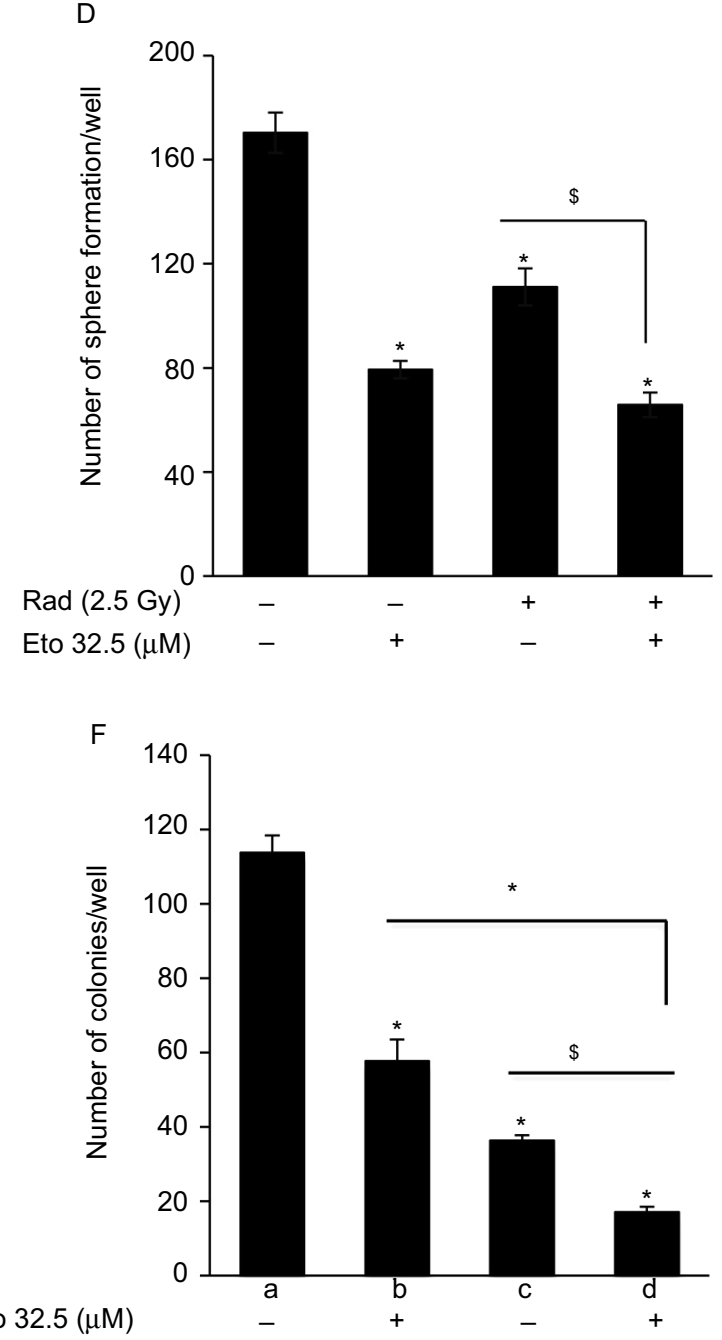

Eto $32.5(\mu \mathrm{M})$ plus etomoxir treated) were dissociated by brief trypsinization

At the end of seventh day, number of clones counted in each group
500 cells seeded in

six-well plates in complete media

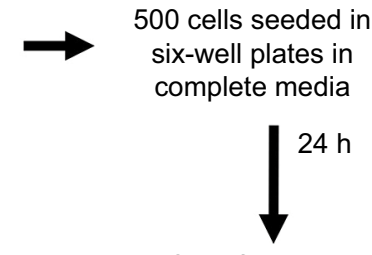

Cells from etomoxir treatment groups retreated with etomoxir $(32.5 \mu \mathrm{M})$

Figure I Etomoxir combination improves radiation efficacy against sphere formation by $\mathrm{H} 460$ lung epithelial cancer cells.

Notes: (A) Experimental scheme. (B) At the end of experiment (day 6), sphere images were captured and representative images are presented (I00x). (C) Average sphere area presented as mean $\pm S E M$. (D) Number of spheres in each group presented as mean \pm SEM. (E) Experimental scheme. (F) At the end of 7 days, number of colonies with $\geq 50$ cells were counted and presented as mean \pm SEM in the bar diagram. In the bar diagram, a represents control group, b represents etomoxir alone, $c$ represents radiation alone, and $d$ represents radiation plus etomoxir.* $p<0.001 ;{ }^{\$} p<0.01 ; " \beta<0.05$.

Abbreviations: Eto, etomoxir; Rad, radiation; SEM, standard error of the mean. 
$1 \times$ PBS $+0.3 \%$ Triton X-100) followed by blocking in 5\% bovine serum albumin (BSA) block buffer (PBS $+0.3 \%$ Triton X-100+5\% BSA). Spheres were then incubated with fluorescein isothiocyanate-conjugated monoclonal antibody against pimonidazole for overnight and mounted with gold antifade reagent with DAPI. Stained spheres images were captured at $200 \times$ magnification using a Nikon D-Eclipse C1 confocal microscope and analyzed using EZ-C1 Free viewer.

\section{Confocal imaging}

Spheres were collected, transferred to eight-well chamber slides coated with matrigel, and permeabilized as above. Thereafter, blocking was performed in 5\% BSA block buffer $(\mathrm{PBS}+0.3 \%$ Triton X-100 + 5\% BSA). Next, spheres were incubated with respective primary antibodies, Ki-67 (1:100), cyclin D1 (1:200), CD44 (1:100), or CPT1A (1:200) in PBST (with 1\% BSA) overnight in a humidified chamber. Thereafter, spheres were incubated with Alexa-Fluor 488 or 594 secondary antibody $(1: 250)$ with DAPI $(1: 1,000)$ for $1 \mathrm{~h}$ and mounted with prolong gold antifade reagent with DAPI. Stained spheres were imaged using a Nikon D-Eclipse C1 confocal microscope and analyzed using EZ-C1 free viewer software. $Z$ stacking was done by complete scanning of spheres in depth, and then a reference point was selected in middle where scans of $5 \mu \mathrm{m}$ interval were taken in both directions. The fluorescence intensity was quantified using ImageJ software (NIH, Bethesda, MD, USA).

\section{Statistical analysis}

Statistical analysis was performed using SigmaStat 2.03 (Jandel Scientific, San Rafael, CA, USA). Data were analyzed by one-way analysis of variance (ANOVA) (Tukey test) and statistically significant differences were considered at $p<0.05$.

\section{Results}

\section{Etomoxir enhances radiation cytotoxicity}

Lung adenocarcinoma $\mathrm{H} 460$ cells were cultured as spheres to serve as a three-dimensional (3D) model to study hypoxia and effect of radiation and etomoxir treatment. Spheres were irradiated one time with a 2.5 Gy dose (experiment day 0 ) followed by treatment with vehicle (dimethyl sulfoxide [DMSO], $0.1 \%)$ or etomoxir $(32.5 \mu \mathrm{M})$. Thereafter, spheres were treated with DMSO or etomoxir on experiment days 2 and 4 (Figure 1A). At the end of the sixth day, sphere number and average sphere area were determined. As shown in Figure 1B and C, radiation exposure reduced the average $\mathrm{H} 460$ sphere area by $67.87 \%(p<0.001)$, and etomoxir combination further reduced the sphere area by $71.04 \%(p<0.001)$ compared with control.
Etomoxir alone treatment reduced the sphere area by $47.06 \%$ $(p<0.001)$. Further, radiation treatment reduced the $\mathrm{H} 460$ sphere number by $34.78 \%(p<0.001)$, while combination with etomoxir reduced the sphere number by $61.37 \%(p<0.001)$ compared with control; etomoxir alone treatment reduced the sphere number by $53.41 \%$ ( $p<0.001$; Figure 1D).

Next, we assessed the colony forming ability of spheres after completion of treatments (vehicle, radiation, etomoxir, or radiation plus etomoxir). In the clonogenic assay, only etomoxir treatment was replenished (wherever mentioned) for continuous inhibition of $\beta$-oxidation (experimental scheme shown in Figure 1E). The etomoxir alone-treated single cells of spheres showed reduced capacity to form colonies by $49.27 \%(p<0.001)$, while irradiated ( $2.5 \mathrm{~Gy})$ sphere cells showed reduced clonogenic potential by $68.04 \%$ $(p<0.001)$. The combination of radiation and etomoxir further inhibited the clonogenic potential of cells from spheres by $85.05 \%(p<0.001)$, and the combined inhibitory effect was significantly better than either radiation or etomoxir alone (Figure 1F). These results further suggested the inhibition of $\beta$-oxidation by etomoxir could improve the cytotoxic effect of radiation against cancer cells.

\section{Etomoxir combination with radiation reduces hypoxic areas}

Next, we examined the effect of etomoxir treatment along with radiation on hypoxia by staining spheres with pimonidazole. Pimonidazole, a hypoxia marker, is a 2-nitroimidazole compound which forms covalent bond with peptide thiols at oxygen levels below $1.3 \%$ and can visualize poorly oxygenated regions in histological samples. ${ }^{20,21}$ Spheres were incubated with pimonidazole $(200 \mu \mathrm{M})$ for $2 \mathrm{~h}$ and then processed as described in the "Methods" section. As shown in Figure 2, hypoxic areas were present in spheres treated with radiation alone; however, combination of etomoxir with radiation reduced the hypoxic areas by 59\% compared with control spheres. The radiation and etomoxir combination effect was significantly better than either radiation or etomoxir alone (Figure 2).

\section{Etomoxir combination with radiation reduces the expression of proliferation, stemness, and $\beta$-oxidation biomarkers}

H460 spheres treated with radiation alone showed higher Ki-67 expression compared with control; whereas, spheres treated with etomoxir $(32.5 \mu \mathrm{M})$ in combination with radiation showed reduced $\mathrm{Ki}-67$ expression compared with radiation treatment alone (Figure $3 \mathrm{~A}$ and quantification 
H460 spheres
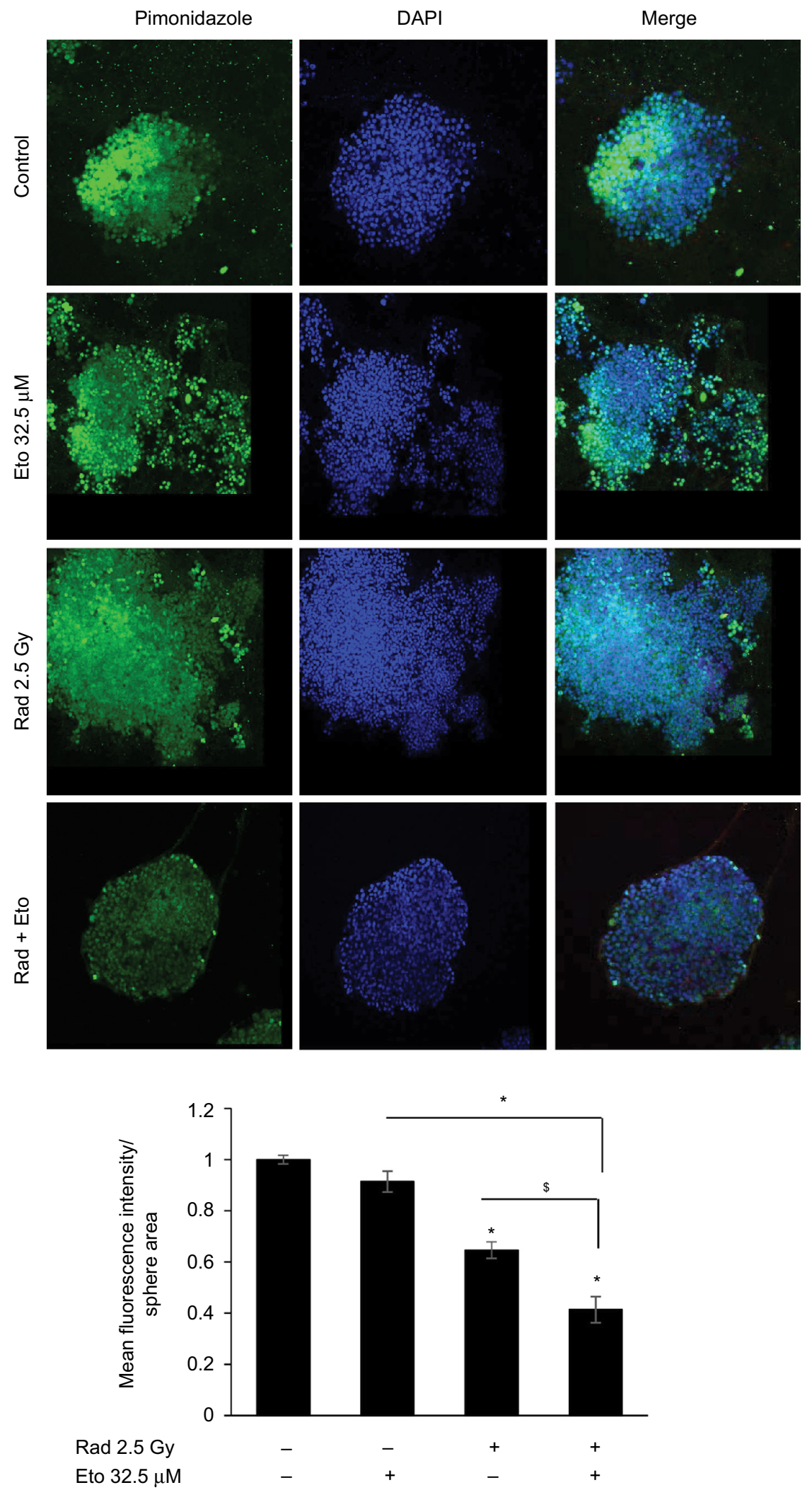

Figure 2 Etomoxir treatment following radiation exposure reduces hypoxic areas in $\mathrm{H} 460$ spheres.

Notes: $\mathrm{H} 460$ spheres were irradiated $(2.5 \mathrm{~Gy})$ and then treated with etomoxir $32.5 \mu \mathrm{M}$. Spheres were re-treated with etomoxir on experiment day 2 and 4 . At the end of day 6, H460 spheres were treated with pimonidazole $(200 \mu \mathrm{M})$ for $2 \mathrm{~h}$ and then processed for immunofluorescence. Representative confocal images are shown (at $200 \times$ magnification). The bar diagram represents the mean fluorescence intensity per unit sphere area as mean \pm SEM. ${ }^{*} p<0.00 \mathrm{I} ;{ }^{\$} p<0.0 \mathrm{I}$.

Abbreviations: DAPI, 4',6-diamidino-2-phenylindole; Eto, etomoxir; Rad, radiation; SEM, standard error of the mean. 


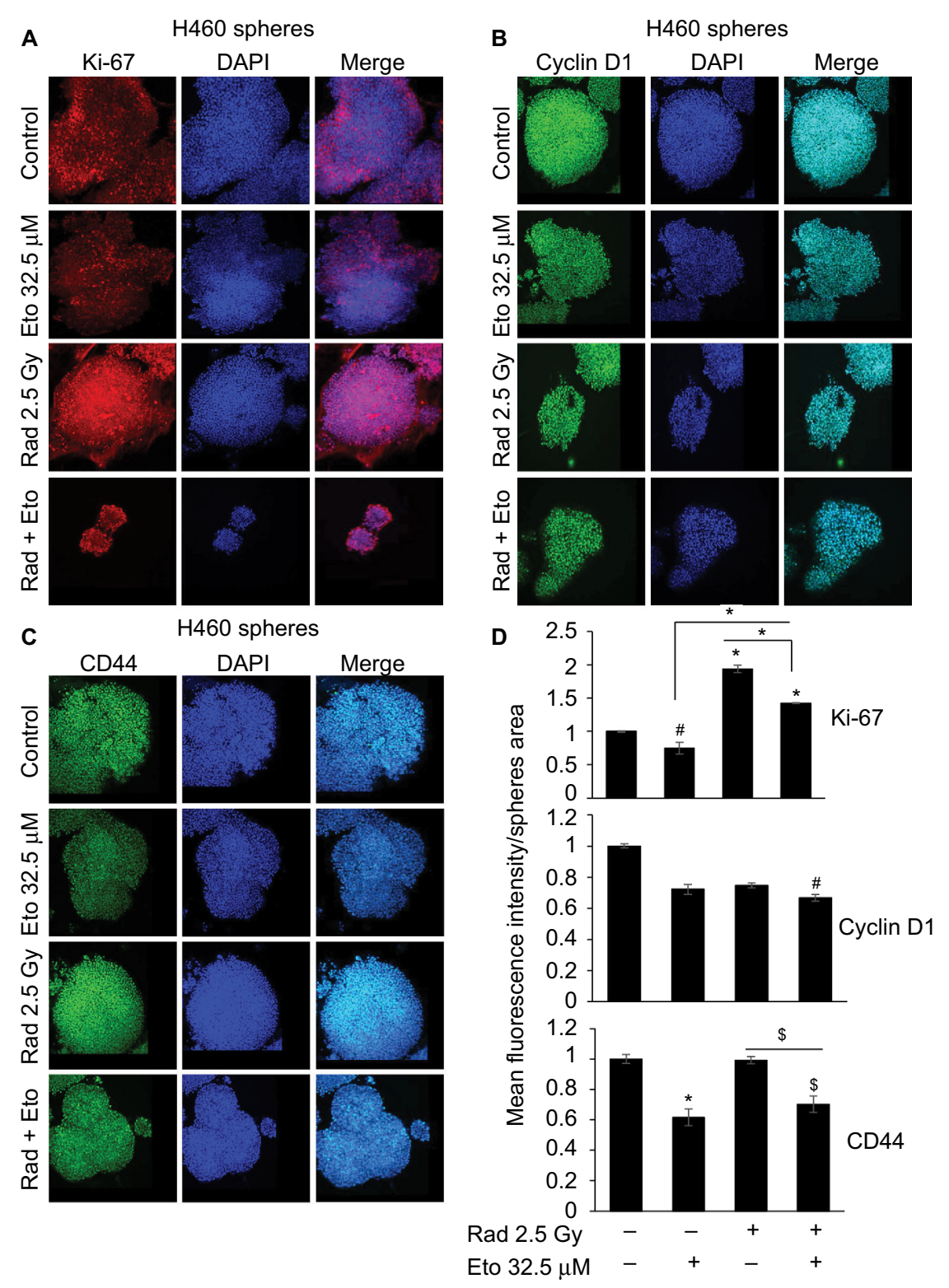

Figure 3 Etomoxir treatment following radiation exposure reduces the expression of proliferation and stemness biomarkers in $\mathrm{H} 460$ spheres.

Notes: (A-C) $5 \times 10^{3} \mathrm{H} 460$ cells were plated in six-well ultralow attachment plates for sphere formation for 6 days. Thereafter, spheres were irradiated with a dose of 2.5 $\mathrm{Gy}$ and then treated with etomoxir $32.5 \mu \mathrm{M}$. Spheres were retreated with etomoxir on experiment days 2 and 4 . At the end of day 6 after first etomoxir treatment, $\mathrm{H} 460$ spheres were processed to analyze (A) Ki-67, (B) cyclin DI, and (C) CD44 expression by immunofluorescence as described in the "Methods" section. Representative images are shown (at 200× magnification). (D) The bar diagram represents the mean fluorescence intensity per unit sphere area for Ki-67, cyclin DI, and CD44 as mean \pm SEM. ${ }^{*} p<0.001 ;{ }^{\$} p<0.01 ;{ }^{*} p<0.05$.

Abbreviations: DAPI, 4',6-diamidino-2-phenylindole; Eto, etomoxir; Rad, radiation; SEM, standard error of the mean.

shown in Figure 3D). Cyclin D1 fluorescence intensity was not significantly affected by etomoxir or radiation alone treatment but the combination showed a significant decrease in cyclin D1 level compared with control (Figure 3B and quantification shown in Figure 3D). Further, etomoxir alone treatment reduced the level of CD44 in H460 spheres; however, radiation alone treatment did not affect the CD44 expression (Figure 3C). The combination of etomoxir and radiation treatment reduced the $\mathrm{CD} 44$ expression compared with radiation treatment alone (Figure $3 \mathrm{C}$ and quantification shown in Figure 3D).

As expected, etomoxir treatment alone strongly reduced the CPT1A expression (a biomarker for $\beta$-oxidation) in spheres (Figure 4). However, spheres treated with radiation alone showed higher CPT1A expression (Figure 4). Importantly, the combination of etomoxir with radiation effectively reduced the CPT1A expression compared with the radiation alone group (Figure 4). 
H460 spheres
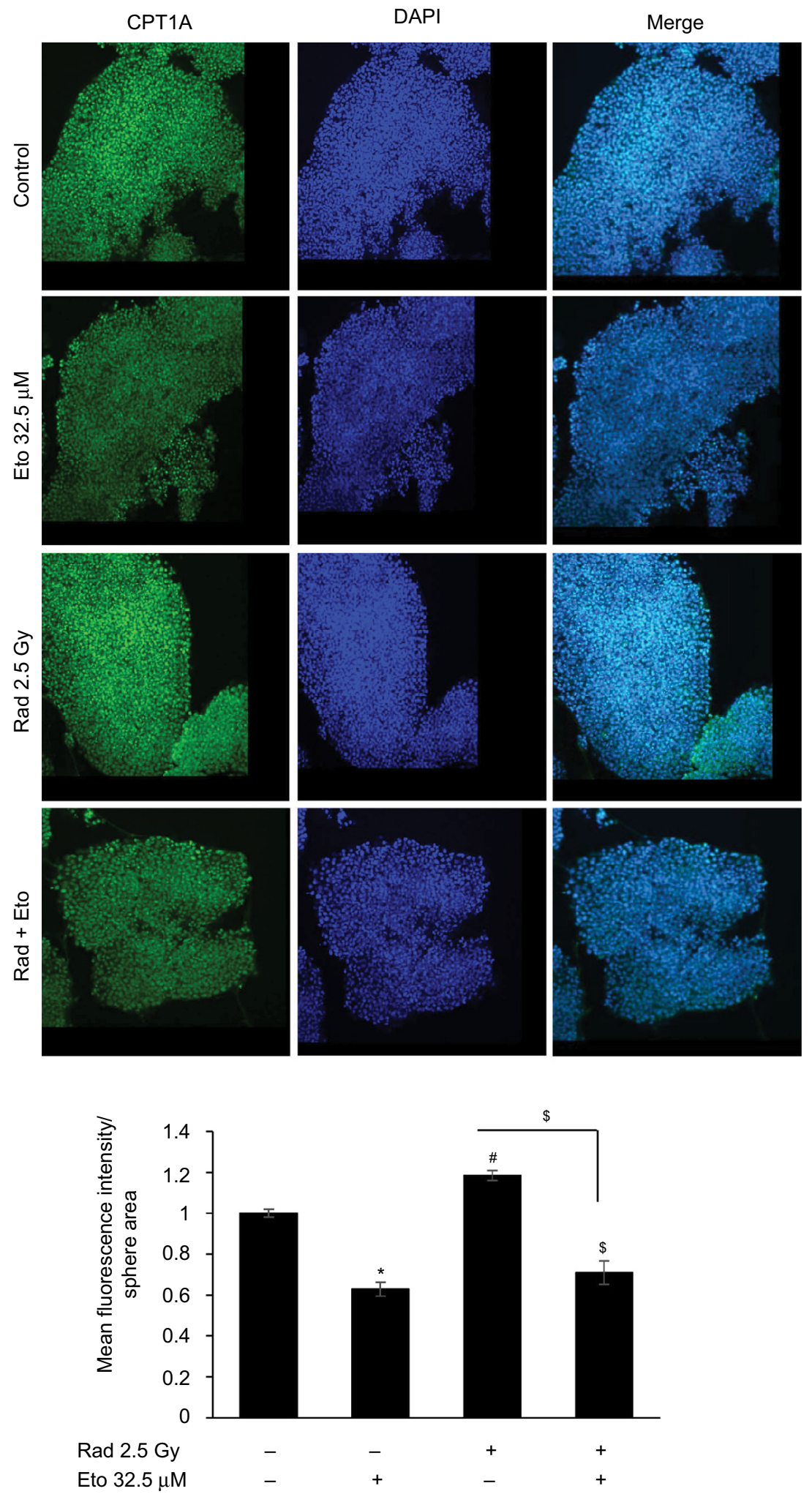

Figure 4 Etomoxir treatment following radiation exposure reduces CPTI expression in $\mathrm{H} 460$ spheres.

Notes: $5 \times 10^{3} \mathrm{H} 460$ cells were plated in six-well ultralow attachment plates for sphere formation for 6 days. Thereafter, spheres were irradiated with a dose of $2.5 \mathrm{~Gy}$ and then treated with etomoxir $32.5 \mu \mathrm{M}$. Spheres were retreated with etomoxir on experiment days 2 and 4 . At the end of day 6 after first etomoxir treatment, H460 spheres were processed to analyze CPTIA expression by immunofluorescence as described in the "Methods" section. Representative images are shown (at 200× magnification). The bar diagram represents the mean fluorescence intensity per unit sphere area as mean \pm SEM. ${ }^{*} p<0.00 \mathrm{I} ;{ }^{\$} p<0.0 \mathrm{I}$.

Abbreviations: CPTIA, carnitine palmitoyltransferase IA; DAPI, 4',6-diamidino-2-phenylindole; Eto, etomoxir; Rad, radiation; SEM, standard error of the mean. 


\section{Radiation treatment effectively reduces sphere size and hypoxic areas in CPTIA knockdown LNCaP cells}

Subsequently, we confirmed the efficacy of radiation and etomoxir combination to reduce sphere growth in human PCa LNCaP cells. As shown in Figure 5A, radiation alone reduced the sphere number by $39.2 \%(p<0.05)$, while the combination of radiation and etomoxir reduced the LNCaP sphere number by $47.2 \%(p<0.01)$. Etomoxir alone treatment reduced the $\mathrm{LNCaP}$ sphere number by $28 \%$ (Figure $5 \mathrm{~A}$ ). Next, we prepared single-cell suspension of spheres from different treatment groups and seeded in ultralow attachment plates to generate second generation spheres. In the second generation spheres, only etomoxir treatment was continued for effective inhibition of $\beta$-oxidation. The etomoxir alone treatment inhibited the second generation sphere numbers by $42.4 \%$ $(p<0.001)$. The total number of second generation spheres was reduced by $76.8 \%(p<0.001)$ in the radiation only treatment group, and combination of radiation and etomoxir showed $89.3 \%$ decrease in the sphere formation ( $p<0.001$; Figure $5 \mathrm{~B}$ ).

To further assess CPT1A role, we performed a similar experiment as above in $\mathrm{LNCaP}$ cells with stable CPT1A knockdown (LNCaP CPT1A KD). Radiation treatment reduced the sphere number and size in both $\mathrm{LNCaP}$ vector control and LNCaP CPT1A KD cells (Figure 5C and D). Interestingly, pimonidazole staining showed that hypoxic areas were significantly reduced following radiation treatment in LNCaP CPT1A KD cells (Figure 5E).

\section{Discussion}

Cancer is an extremely complex disease where in addition to several genetic and epigenetic factors, various tumor microenvironment components also affect cancer development and progression. Hypoxia in tumor microenvironment is one such major factor that appears to correlate with tumor growth, progression, and relapse. A variety of approaches have been used to target tumor hypoxia, which include hyperbaric oxygenation (HBO), accelerated radiotherapy with carbogen and nicotinamide (ARCON) (a combination of carbogen breathing and nicotinamide), anemia correction, and antiangiogenic therapy, thereby increasing plasma and tissue oxygen level. ${ }^{22,23}$ However, so far, these therapies have not shown significant efficacy in clinic. For example, the effect of HBO is transient and diminishes in minutes, and the pressure is not tolerated by several patients. ${ }^{23}$ Similarly, clinical trials with inhibitors of HIF and hypoxia-induced signaling such as PI3K, Akt, and mTOR inhibitors have not shown promising results owing to poor tissue penetration to reach hypoxic region as well as pharmacokinetics and pharmacodynamics properties of the tested drugs. ${ }^{1024}$ These clinical outcomes have warranted testing additional novel and innovative measures to target hypoxic cells in tumors.

The reprogramming of lipid metabolism in tumor is now being recognized as an important event for tumor cell growth and progression. ${ }^{25}$ Cancer cells fulfill their elevated needs of lipids including fatty acids and phospholipids by de novo lipogenesis. ${ }^{26,27}$ Cellular lipids also help in survival and proliferation of cancer cells under hypoxia. ${ }^{18,28,29}$ We have recently reported that lipid oxidation is important following reoxygenation in the survival and increased proliferation of hypoxic PCa cells. ${ }^{19} \mathrm{CPT} 1$ is a major regulator of fatty acid oxidation, which translocates fatty acids conjugated with carnitine to the mitochondria where they can be oxidized to produce acetyl$\mathrm{CoA}$, which then goes to Krebs cycle and produces NADH and FADH2 for oxidative phosphorylation. Hence, targeting CPT1 can inhibit cancer cell growth by limiting the energy supply of the cell. Currently, several CPT1 inhibitors are in clinical use/ trials for treatment of heart disease $\mathrm{e}^{30}$ and could be potentially useful for combination cancer therapy along with radiation. We reported that CPT1 knockdown or inhibition by etomoxir makes hypoxia-reoxygenated cancer cells sensitive toward growth inhibition. ${ }^{19}$ Results from the present study further showed that etomoxir combination could improve the anticancer efficacy of radiation and reduce the hypoxic areas in spheres.

In the present study, we employed a sphere model to target physiological hypoxia by single fraction radiation and etomoxir. This model is currently widely used to determine the stemness of cancer cells in in vitro conditions. ${ }^{31-33}$ The core region of fully grown spheres is less oxygenated and offers a useful model to understand the hypoxia-mediated biological effects such as radioresistance. We confirmed the extent of hypoxia in spheres using pimonidazole, a nitroimidazole compound considered more sensitive than the microelectrode method of oxygen concentration measurement. ${ }^{34}$ As expected, hypoxic regions stained with pimonidazole were located primarily in the irradiated spheres, and etomoxir combination reduced the pimonidazole-stained areas. This combination also reduced the expression of proliferation and stemness biomarkers, as well as decreased the CPT1 expression in the spheres. The increased sensitivity to radiation in combination with etomoxir could be through inhibition of fatty acid oxidation in reoxygenated cells resulting in reduced proliferation and stemness. Alternatively, the reduced pAKT observed in CPT1 $\mathrm{KD}$ cells ${ }^{35}$ could be responsible for increased sensitivity to the radiation treatment. These results suggest CPT1 as a novel target to overcome radioresistance 
A

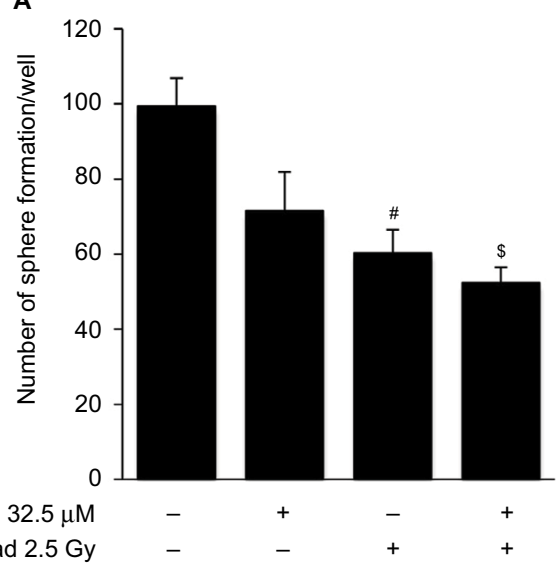

Eto $32.5 \mu \mathrm{M}$ $\operatorname{Rad} 2.5 \mathrm{~Gy}$

C

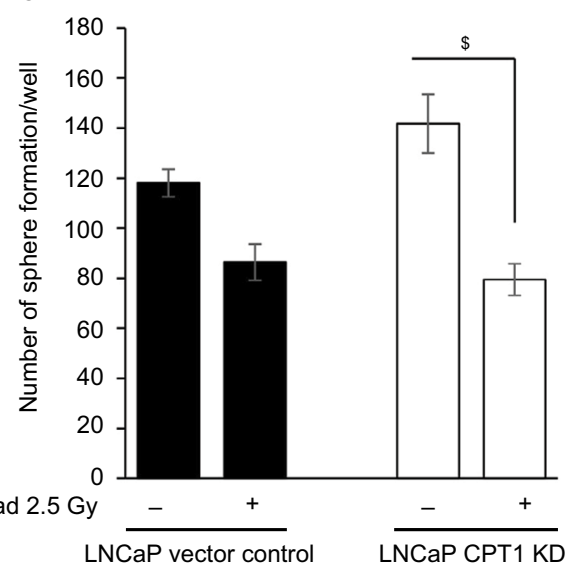

B

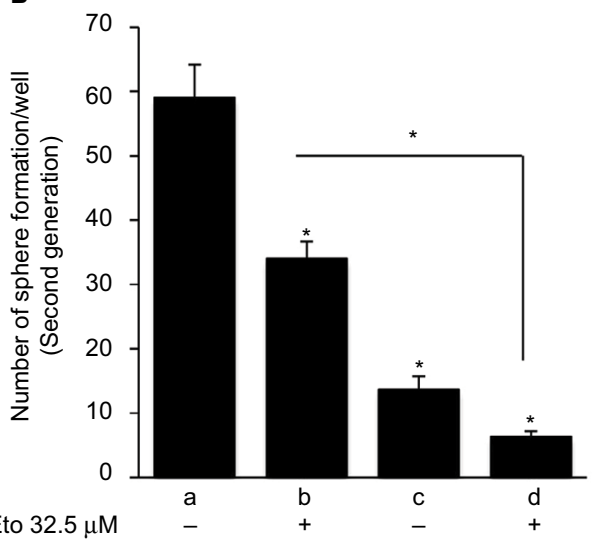

D

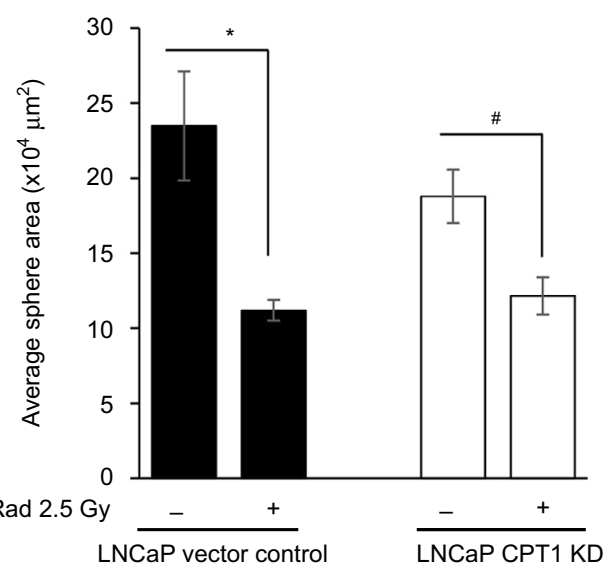

E

LNCaP CPT1 KD spheres, day 6
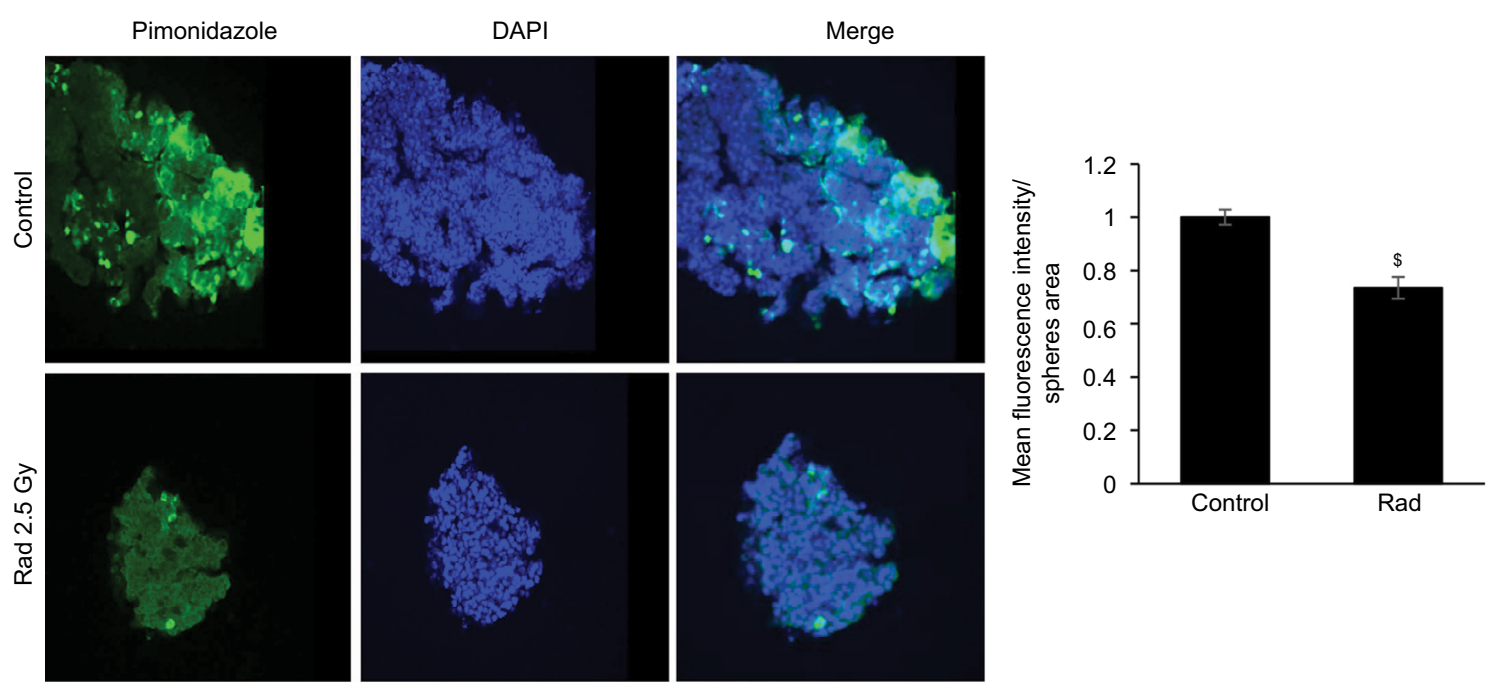

Figure 5 Radiation treatment effectively reduces sphere size and hypoxic areas in CPTIA knockdown LNCaP cells.

Notes: $\mathrm{LNCaP}$ spheres were irradiated (2.5 Gy) and then treated with etomoxir $32.5 \mu \mathrm{M}$. At the end of day 6, LNCaP spheres number was counted and presented as mean \pm SEM in the bar diagram (A). Thereafter, spheres in each group were dissociated into single cells and plated I,000 cells/well in ultralow attachment six-well plates. Cells were treated with etomoxir $32.5 \mu \mathrm{M}$ after $24 \mathrm{~h}$ of seeding and every $48 \mathrm{~h}$ thereafter. Number of spheres were counted after day 6 of treatment and presented as mean \pm SEM in the bar diagram (B). In the bar diagram, a represents control group, b represents etomoxir alone, c represents radiation alone, and $d$ represents radiation plus etomoxir. (C, D) Vector control LNCaP cells and LNCaP CPTIA KD cells were cultured to form spheres and then treated with radiation (2.5 Gy). After 6 days, sphere number and area were measured and presented as mean \pm SEM in the bar diagrams. (E) LNCaP CPTI KD spheres were treated with $200 \mu M$ of pimonidazole for $2 \mathrm{~h}$ and processed for immunofluorescence. Representative confocal images are shown (at 200× magnification). The bar diagram represents the mean fluorescence intensity per unit sphere area as mean \pm SEM. ${ }^{*} p<0.001 ;{ }^{\$} p<0.01 ; " p<0.05$.

Abbreviations: CPTIA, carnitine palmitoyltransferase IA; DAPI, 4',6-diamidino-2-phenylindole; Eto, etomoxir; KD, knockdown; Rad, radiation; SEM, standard error of the mean. 


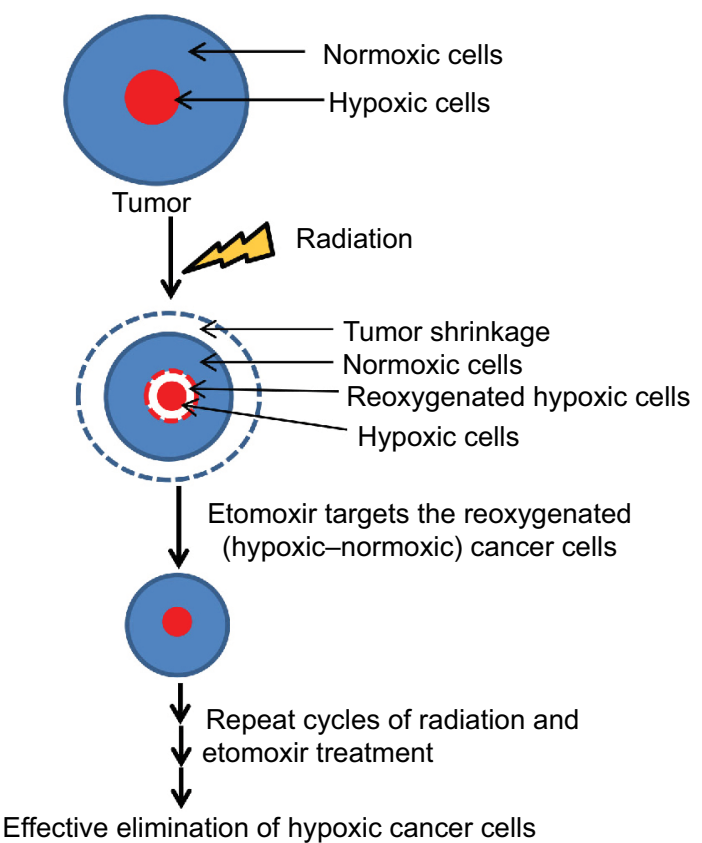

Figure 6 Proposed hypothesis.

Note: Etomoxir, an inhibitor of $\beta$-oxidation, treatment following radiation exposure could effectively eliminate hypoxic cells in the tumor, inhibiting tumor growth and preventing disease relapse.

in tumors. The limitation of this model is that spheres are enriched in stem-like cells and might not represent the bulk cancer cells present in solid tumors. However, the success of the radiation and etomoxir against stem cell-enriched spheres, which are relatively difficult to target, further underscores the value of this combination. Furthermore, the applicability of these results to the clinic is much feasible, since clinically approved CPT1 inhibitors like perhexiline or partial $\beta$-oxidation inhibitors like ranolazine ${ }^{36}$ could be administered to patients concomitantly with their radiation treatment or immediately after.

\section{Conclusion}

Results from the present study suggest that combining $\beta$-oxidation inhibitor etomoxir with radiation could be a novel and effective strategy for reducing hypoxia in solid tumors (depicted in Figure 6), thereby reducing chances of disease relapse.

\section{Data sharing statement}

The datasets analyzed during the current study are available from the corresponding author on reasonable request.

\section{Acknowledgments}

We thank Dr Barbara Frederick for her helpful inputs in this project.We acknowledge the grant support from Colorado
Clinical and Translational Science Institute and NCI R21 CA199628 (to GD), K01 CA168934 (to IRS), and R01 CA102514 (to RA).

\section{Author contributions}

$\mathrm{AD}$ performed the experiments and wrote the manuscript; CA contributed in cell culture and manuscript writing; IRS, $\mathrm{DR}, \mathrm{RS}$, and RA provided the reagents and contributed in experimental design and manuscript writing; and GD developed the original hypothesis, study design, supervised the experiments, provided reagents, and contributed in manuscript writing. All authors contributed toward data analysis, drafting and revising the paper and agree to be accountable for all aspects of the work.

\section{Disclosure}

The authors report no conflicts of interest in this work.

\section{References}

1. Deep G PG. Hypoxia-induced signaling promotes prostate cancer progression: Exosomes role as messenger of hypoxic response in tumor microenvironment. Crit Rev Oncog. 2015;20(5-6):419-434.

2. Hung JJ, Yang MH, Hsu HS, Hsu WH, Liu JS, Wu KJ. Prognostic significance of hypoxia-inducible factor-1alpha, TWIST1 and Snail expression in resectable non-small cell lung cancer. Thorax. 2009;64(12):1082-1089.

3. Turaka A, Buyyounouski MK, Hanlon AL, Horwitz EM, Greenberg RE, Movsas B. Hypoxic prostate/muscle PO2 ratio predicts for outcome in patients with localized prostate cancer: long-term results. Int J Radiat Oncol Biol Phys. 2012;82(3):e433-e439.

4. Milosevic M, Warde P, Menard C, et al. Tumor hypoxia predicts biochemical failure following radiotherapy for clinically localized prostate cancer. Clin Cancer Res. 2012;18(7):2108-2114.

5. Brustugun OT. Hypoxia as a cause of treatment failure in non-small cell carcinoma of the lung. Semin Radiat Oncol. 2015;25(2):87-92.

6. Lee S-1-o, Ryu H, Son Ar, et al. TGF- $\beta$ and Hypoxia/Reoxygenation promote radioresistance of A549 lung cancer cells through activation of Nrf2 and EGFR. Oxid Med Cell Longev. 2016;2016:6823471.

7. Marampon F, Gravina GL, Zani BM, et al. Hypoxia sustains glioblastoma radioresistance through ERKs/DNA-PKcs/HIF-1alpha functional interplay. Int J Oncol. 2014;44(6):2121-2131.

8. Sheehan JP, Shaffrey ME, Gupta B, Larner J, Rich JN, Park DM. Improving the radiosensitivity of radioresistant and hypoxic glioblastoma. Future Oncol. 2010;6(10):1591-1601.

9. Li JZ, Gao W, Chan JY-W, Ho W-K, Wong T-S. Hypoxia in head and neck squamous cell carcinoma. ISRN Otolaryngology. 2012;2012:8.

10. Wigerup C, Påhlman S, Bexell D. Therapeutic targeting of hypoxia and hypoxia-inducible factors in cancer. Pharmacol Ther. 2016;164:152-169.

11. Moeller BJ, Cao Y, Li CY, Dewhirst MW. Radiation activates HIF-1 to regulate vascular radiosensitivity in tumors: role of reoxygenation, free radicals, and stress granules. Cancer Cell. 2004;5(5):429-441.

12. Pires IM, Olcina MM, Anbalagan S, et al. Targeting radiation-resistant hypoxic tumour cells through ATR inhibition. Br J Cancer. 2012; 107(2):291-299.

13. Ward PS, Thompson CB. Metabolic reprogramming: a cancer hallmark even Warburg did not anticipate. Cancer Cell. 2012;21(3):297-308.

14. Romero-Garcia S, Lopez-Gonzalez JS, B'ez-Viveros JL, AguilarCazares D, Prado-Garcia H. Tumor cell metabolism. Cancer Biol Ther:2011;12(11):939-948. 
15. Bensaad K, Favaro E, Lewis CA, et al. Fatty acid uptake and lipid storage induced by HIF-1alpha contribute to cell growth and survival after hypoxia-reoxygenation. Cell Rep. 2014;9(1):349-365.

16. Huang D, Li T, Li X, et al. HIF-1-mediated suppression of acyl-CoA dehydrogenases and fatty acid oxidation is critical for cancer progression. Cell Rep. 2014;8(6):1930-1942.

17. Metallo CM, Gameiro PA, Bell EL, et al. Reductive glutamine metabolism by IDH1 mediates lipogenesis under hypoxia. Nature. 2012;481(7381): 380-384.

18. Sun RC, Denko NC. Hypoxic regulation of glutamine metabolism through HIF1 and SIAH2 supports lipid synthesis that is necessary for tumor growth. Cell Metab. 2014;19(2):285-292.

19. Schlaepfer IR, Nambiar DK, Ramteke A, et al. Hypoxia induces triglycerides accumulation in prostate cancer cells and extracellular vesicles supporting growth and invasiveness following reoxygenation. Oncotarget. 2015;6(26):22836-22856.

20. Gross MW, Karbach U, Groebe K, Franko AJ, Mueller-Klieser W. Calibration of misonidazole labeling by simultaneous measurement of oxygen tension and labeling density in multicellular spheroids. Int $J$ Cancer. 1995;61(4):567-573.

21. Kizaka-Kondoh S, Konse-Nagasawa H. Significance of nitroimidazole compounds and hypoxia-inducible factor-1 for imaging tumor hypoxia. Cancer Sci. 2009;100(8):1366-1373.

22. Moen I, Stuhr LEB. Hyperbaric oxygen therapy and cancer-a review. Target Oncol. 2012;7(4):233-242.

23. Baumann R, Depping R, Delaperriere M, Dunst J. Targeting hypoxia to overcome radiation resistance in head \& neck cancers: real challenge or clinical fairytale? Expert Rev Anticancer Ther. 2016;16(7):751-758

24. Saggar JK, Yu M, Tan Q, Tannock IF. The tumor microenvironment and strategies to improve drug distribution. Front Oncol. 2013;3:154.

25. Beloribi-Djefaflia S, Vasseur S, Guillaumond F. Lipid metabolic reprogramming in cancer cells. Oncogenesis. 2016;5:e189.
26. Zaidi N, Lupien L, Kuemmerle NB, Kinlaw WB, Swinnen JV, Smans K. Lipogenesis and lipolysis: the pathways exploited by the cancer cells to acquire fatty acids. Prog Lipid Res. 2013;52(4):585-589.

27. Baenke F, Peck B, Miess H, Schulze A. Hooked on fat: the role of lipid synthesis in cancer metabolism and tumour development. Dis Model Mech. 2013;6(6):1353-1363.

28. Kamphorst JJ, Chung MK, Fan J, Rabinowitz JD. Quantitative analysis of acetyl-CoA production in hypoxic cancer cells reveals substantial contribution from acetate. Cancer Metab. 2014;2:23.

29. Eales KL, Hollinshead KER, Tennant DA. Hypoxia and metabolic adaptation of cancer cells. Oncogenesis. 2016;5:e190.

30. Lionetti V, Stanley WC, Recchia FA. Modulating fatty acid oxidation in heart failure. Cardiovasc Res. 2011;90(2):202-209.

31. Deep G, Jain AK, Ramteke A, et al. SNAI1 is critical for the aggressiveness of prostate cancer cells with low E-cadherin. Mol Cancer 2014;13(1):37

32. Bar EE, Lin A, Mahairaki V, Matsui W, Eberhart CG. Hypoxia Increases the Expression of Stem-Cell Markers and Promotes Clonogenicity in Glioblastoma Neurospheres. Am J Pathol. 2010;177(3) 1491-1502.

33. Qiang L, Wu T, Zhang HW, et al. HIF-1[alpha] is critical for hypoxiamediated maintenance of glioblastoma stem cells by activating Notch signaling pathway. Cell Death Differ. 2012;19(2):284-294.

34. Raleigh JA, Chou SC, Arteel GE, Horsman MR. Comparisons among pimonidazole binding, oxygen electrode measurements, and radiation response in C3H mouse tumors. Radiat Res. 1999;151(5):580-589.

35. Schlaepfer IR, Rider L, Rodrigues LU, et al. Lipid catabolism via CPT1 as a therapeutic target for prostate cancer. Mol Cancer Ther. 2014;13(10):2361-2371.

36. Samudio I, Harmancey R, Fiegl M, et al. Pharmacologic inhibition of fatty acid oxidation sensitizes human leukemia cells to apoptosis induction. J Clin Invest. 2010;120(1):142-156.

\section{Hypoxia}

\section{Publish your work in this journal}

Hypoxia is an international, peer-reviewed, open access journal that aims to improve understanding of the biological response to hypoxia. The journal will publish original research articles, reviews, methodological advances, clinical studies, and expert opinions that identify developments in the regulation of the physiological and pathological responses to
Submit your manuscript here: https://www.dovepress.com/hypoxia-journal

\section{Dovepress}

hypoxia and in the therapeutic targeting of hypoxia-responsive pathways. The manuscript management system is completely online and includes a very quick and fair peer-review system, which is all easy to use. Visit http://www.dovepress.com/testimonials.php to read real quotes from published authors. 